\title{
Prognostic impact of pretreatment FDG PET parameters in locally advanced cervical cancer treated with concomitant chemoradiotherapy: A systematic review and meta-analysis
}

\author{
Lu Han ${ }^{1}$, Qi Wang ${ }^{2}$, Lanbo Zhao ${ }^{3}$, Kailu Zhang ${ }^{1}$, Yiran Wang ${ }^{1}$, Xue Feng ${ }^{1}$, Yuliang Zou ${ }^{2}$, \\ and Qiling $\mathrm{Li}^{4}$ \\ ${ }^{1}$ the First Affiliated Hospital of Xi'an Jiaotong University \\ ${ }^{2}$ Department of Obstetrics and Gynecology, First Affiliated Hospital, Xi'an Jiaotong \\ University, Xi'an, Shaanxi, China \\ ${ }^{3} \mathrm{Xi}$ 'an Jiaotong University \\ ${ }^{4}$ Xian Jiaotong University
}

October 16, 2020

\begin{abstract}
Background More evidence has shown FDG PET/CT parameters might be associated with survival of patients with locally advanced cervical cancer (LACC) treated with concurrent chemo-radiotherapy (CCRT). Objectives To perform this metaanalysis to investigate the prognostic value of pretreatment FDG PET/CT parameters. Search strategy PubMed and Embase. Selection criteria Studies that met the following criteria were enrolled: patients treated with CCRT for LACC; FDG PET/CT scans performed before or during treatment; and relationship between the parameters of FDG PET/CT and the prognosis of patients were investigated. Data collection and analysis Pooled hazard ratios (HRs) with 95\% confidence intervals (CIs) were used to estimate overall survival (OS) or event-free survival (EFS). Main results Higher primary tumor TLG (HR $=1.843,95 \%$ $\mathrm{CI}=1.100-3.086, \mathrm{P}=0.02)$ and $\mathrm{MTV}(\mathrm{HR}=2.06,95 \% \mathrm{CI}, 1.21-3.51, \mathrm{P}=0.007)$ were significantly associated with shorter EFS. Patients with high SUVmax have a shouter OS than those with low SUVmax (HR = 2.582, 95\% CI 1.936-3.443, P <0.001). Primary tumor SUVmax $(\mathrm{HR}=1.938,95 \% \mathrm{CI}, 1.203-3.054, \mathrm{P}=0.004)$ and nodal SUVmax $(\mathrm{HR}=3.478,95 \% \mathrm{CI}=2.006-$ $6.029, \mathrm{P}<0.001$ ) were significantly correlated with EFS with relatively high heterogeneity ( $\mathrm{I} 2=84 \%$, $\mathrm{I} 2=69.4 \%$, respectively). Nodal SUVmax $(\mathrm{HR}=2.095,95 \% \mathrm{CI}=2.027-2.166, \mathrm{P}<0.001)$ were significantly correlated with OS with relatively high heterogeneity $(\mathrm{I} 2=54.1 \%, \mathrm{P}=0.140$ ). Other parameters had no detected association with survival. Conclusions Pretreatment FDG PET/CT parameters serve as a prognostic predictor.
\end{abstract}

\section{Introduction}

Cervical cancer is a common gynecological malignancy with around 569,847 new cases and 311,365 deaths annually worldwide, and a significant proportion of patients are diagnosed at a locally advanced stage. ${ }^{1}$ For locally advanced cervical cancer (LACC), concurrent chemoradiotherapy (CCRT), using cisplatin-based chemotherapy in association with pelvic external-beam radiotherapy and subsequent brachytherapy is considered the standard treatment. ${ }^{2}$ Although the contribution of CCRT toward an improvement in survival outcomes and reduction in recurrence has been well confirmed, the complete clinical response of these patients is $70 \%-90 \%$, and about one-third of patients with CC experience recurrence. ${ }^{3,4}$

Accurate prediction of recurrence is of great significance. Several traditional clinical and pathological factors are identified as poor prognostic factors, which include advanced International Federation of Gynecology and Obstetrics (FIGO) stage, presence of lymph node metastasis, parametrial invasion, histological tumor 
type, and a large tumor diameter. ${ }^{5-7}$ However, there are still no satisfactory parameters sufficient to predict prognosis accurately.

Computed tomography (CT), magnetic resonance imaging (MRI), and fluorodeoxyglucose positron emission CT (FDG PET/CT) are the most commonly used imaging modalities. Compared to CT or MRI, FDG $\mathrm{PET} / \mathrm{CT}$ could show metabolic information on tumors and more accurate assessment of lymph node involvement, distant metastasis, and recurrent disease. ${ }^{8}$ Therefore, FDG PET/CT has been widely used in staging, therapeutic strategy, and treatment response assessment of patients with CC. ${ }^{9}$

With the technology developed, imaging has provided the potential of a prognostic biomarker. ${ }^{10}$ Except for the aforementioned roles, quantitative parameters derived from FDG-PET/CT, including maximum standardized uptake value $\left(\mathrm{SUV}_{\max }\right)$, average $\mathrm{SUV}$ ( $\left.\mathrm{SUV}_{\text {mean }}\right)$, metabolic tumor volume $(\mathrm{MTV})$, and total lesion glycolysis (TLG), have recently been proposed as prognostic biomarkers for patients with LACC who are treated with CCRT, but the results of studies show some extent differences. For example, Im et al. detected that the patients with high $\mathrm{SUV}_{\max }$ measurement on tumor tissue had a higher risk of recurrence or progression than those with low $\mathrm{SUV}_{\max }(\mathrm{HR}=2.14,95 \% \mathrm{CI}=1.08-4.22, \mathrm{P}=0.029)$, ${ }^{11}$ but Chong et al. draw the opposite conclusion ( $\mathrm{HR}=0.673,95 \% \mathrm{CI}=0.5-4.0, \mathrm{P}=0.412) .{ }^{12}$ Therefore, we performed this metaanalysis to synthetize the relevance of FDG PET/CT parameters as prognostic biomarkers for patients with LACC who are treated with CCRT.

\section{Methods}

\section{Literature search and selection criteria}

Embase and Pubmed (from inception to May 2020) were systematically searched, using the appropriate terminology as described inTable $\mathbf{S 1}$. Besides, the reference lists of the articles reviewed as full texts were also searched manually. The inclusion criteria in the meta-analysis were as follows: patients treated with CCRT for LACC; FDG PET/CT scans performed before or during treatment; and relationship between the parameters of FDG PET/CT and the prognosis of patients was detected. Studies meeting the following criteria were excluded: publication type other than original research articles (i.e., review articles, conference abstracts, and editorial), patients treated with surgery at any point in treatment, patients treated with chemotherapy or radiotherapy alone, and patients lacking important information for analysis (i.e., articles reporting hazard ratios [HRs], using Cox proportional hazards modeling with parameters as a continuous variable). Two authors conducted the searches and screening independently. Any discrepancies were resolved by consensus with another reviewer.

\section{Data extraction}

The following information was extracted using a standardized form: surname of first author, year of publication, country, design (prospective or retrospective), patients' characteristics (including patients' number, tumor node metastasis [TNM] staging, histology, radiotherapy technique, the schedule of systemic therapy, and follow-up period), and outcomes.

\section{Statistical analysis}

The primary outcome, progression event-free survival (EFS), was defined as the date from initiating therapy to recurrence or metastasis. In some included studies, disease-free survival (DFS) or progression-free survival (PFS) was obtained as the primary outcome, but they were all redefined as EFS in this meta-analysis. The secondary outcome was overall survival (OS), defined as the date from initiating therapy until death. The effect sizes of the prognostic values of $\mathrm{SUV}_{\text {max }}, \mathrm{SUV}_{\text {mean }}$, MTV, and TLG were measured in terms of HR. An HR greater than 1 implied worse survival for patients with a high $\mathrm{SUV}_{\max }, \mathrm{SUV}_{\text {mean }}$, MTV, or TLG. The most adjusted estimate of hazard ratio (HR) was extracted directly from each study, if provided by the authors. Otherwise, the HR estimate and its variance were extracted from Kaplan-Meier curves by Engauge Digitizer, version 3.0 (http://digitizer.sourceforge.net). Heterogeneity was assessed with the Q test and $I^{2}$ statistic, and $P$ value less than 0.05 or $I^{2}$ values higher than $50 \%$ indicated significant heterogeneity. The fixed-effects model was used to estimate the cases with homogeneity and the random-effects model was used 
for the cases with significant heterogeneity. Publication bias of the studies was visually displayed by the asymmetry of an inverted funnel plot, and quantitatively evaluated by Egger's tests with the $P$ value less than 0.05 suggesting the significant publication bias. Sensitivity analyses were conducted to assess the stability of the meta-analysis results. All statistical analysis was conducted with STATA 14.0 (STATA Corporation, College Station, TX, USA) software.

\section{Results}

\section{Study selection}

We picked up 721 potentially eligible articles in the electronic search. 78 articles were removed for duplication, and 628 articles were excluded after the reviewers read the titles and abstracts. In total, 14 eligible studies with a total of 1313 patients were included in this meta-analysis after reviewing the full text (Figure 1) .

\section{Patient characteristics and treatment features}

The median age of patients included in the studies was 51.5-58 years (range from 21 to 89 years). Only 12 of the 14 studies provided information about histology, and89.14\% patients had a squamous cell carcinoma (1076/1205)(Table 1) . The largest stage subgroup was FIGO stage IIB, which contained $64.66 \%(846 / 1313)$ of patients. The median follow-up for all patients was 22-60 months (range from 3 to 129 months) (Table 2) . No information on the radiotherapy technique used was available in three studies, and most patients of the other studies were treated with 3D-conformal radiotherapy. The widely adopted external beam radiotherapy regimen consisted of conventional fractionation of 1.8 Gy per fraction (8/9 studies) for a total dose of 45-50.4 Gy. The intracavitary brachytherapy regimen used in majority of patients consisted of fractionation of 7 Gy per fraction (6/10 studies) with four cycles (4/9 studies). The adopted concurrent chemotherapy regimen was standard weekly $40 \mathrm{mg} / \mathrm{m}^{2}$ cisplatin in most patients (10/13 studies) (Table 3 ) .

\section{Primary outcome: EFS}

Night articles about $\mathrm{SUV}_{\max }$ measuring on tumor tissue were included in the study. ${ }^{11-19}$ The combined HR for EFS of higher SUV $\max$ was 1.938 (95\% CI, 1.203-3.054, P = 0.004) (Figure S1A), which meant the patients with high $\mathrm{SUV}_{\max }$ measurement on tumor tissue had a higher risk of recurrence or progression than those with low $\mathrm{SUV}_{\max }$. However, significant heterogeneity existed between the articles $\left(\mathrm{I}^{2}=84 \%, \mathrm{P}=\right.$ 0.000). The publication bias was significant based on the funnel graph (Figure S1B) and Egger's test (P $=0.015)$. The results obtained through sensitivity analysis was relatively stable (Figure S1C).

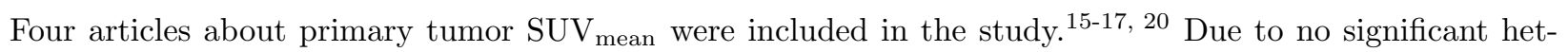
erogeneity $\left(\mathrm{I}^{2}=0 \%, \mathrm{P}=0.938\right)$, the fixed-effects model was performed. The synthesized results showed that patients with high $\mathrm{SUV}_{\text {mean }}$ had a moderately higher risk of recurrence or progression than those with low $\mathrm{SUV}_{\text {mean }}$, but without statistically significant $(\mathrm{HR}=1.182,95 \% \mathrm{CI}=0.899-1.544, \mathrm{P}=0.230)$ (Figure S2A) . Begg's funnel plot and Egger's test indicated no publication bias $(P=0.345)$, and sensitivity analysis showed that the analysis was relatively stable (Figure S2B-C) .

Three articles about MTV, measuring tumor tissue, were included in the study. ${ }^{12,}{ }^{15}, 17$ With no significant heterogeneity $\left(\mathrm{I}^{2}=20.9 \%, \mathrm{P}=0.282\right)$, the combined HR for EFS with higher MTV was 2.06 (95\% CI, 1.21$3.51, \mathrm{P}=0.007$ ) (Figure 2A). It indicated thatpatients with high MTV had a higher risk of recurrence or progression than those with low MTV. Because the data was limited, publication bias was not performed.

Four articles about primary tumor TLG were included in the study. ${ }^{15,} 17,20,21$ In the absence of significant heterogeneity $\left(\mathrm{I}^{2}=0 \%, \mathrm{P}=0.552\right)$, the fixed-effects model was applied. The synthesized results showed that patients with high TLG had a significantly higher risk of recurrence or progression than those with low TLG $(\mathrm{HR}=1.843,95 \% \mathrm{CI}=1.100-3.086, \mathrm{P}=0.02)$ (Figure 2B) . Begg's funnel plot and Egger's test indicated no publication bias $(\mathrm{P}=0.261)$, and sensitivity analysis showed analysis was relatively stable (Figure 2C-D) .

Five articles about nodal SUV $\max$ were included in the study. ${ }^{13,19,21-23}$ For significant heterogeneity $\left(\mathrm{I}^{2}=\right.$ $69.4 \%, \mathrm{P}=0.011)$, the random-effects model was applied. The synthesized results showed that patients 
with higher nodal $\mathrm{SUV}_{\max }$ had a significantly higher risk of recurrence or progression than those with lower nodal $\mathrm{SUV}_{\max }(\mathrm{HR}=3.478,95 \% \mathrm{CI}=2.006-6.029, \mathrm{P}<0.001)$ (Figure S3A). The publication bias was significant based on the funnel graph(Figure S3B) and Egger's test $(\mathrm{P}=0.04)$. Sensitivity analysis proved that the results was relatively stable (Figure S3C) .

\section{Second outcome: OS}

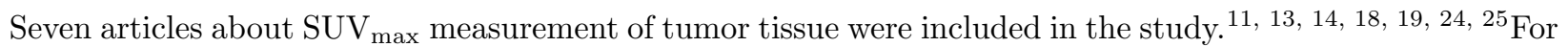
moderate heterogeneity $\left(\mathrm{I}^{2}=36.2 \%, \mathrm{P}=0.151\right)$, the fixed-effects model was applied when pooling HR. The combined HR was 2.582 (95\% CI 1.936-3.443, P <0.001), which meant patients with high $\mathrm{SUV}_{\max }$ had a shorter survival time than those with low $\mathrm{SUV}_{\max }$ (Figure 3A). Begg's funnel plot and Egger's test indicated no publication bias $(\mathrm{P}=0.113)$, and sensitivity analysis showed that the analysis was relatively stable(Figure 3B-C) .

Two articles about primary tumor $\mathrm{SUV}_{\text {mean }}$ were included in the study. ${ }^{14,}{ }^{20}$ For no heterogeneity $\left(\mathrm{I}^{2}=\right.$ $0.0 \%, \mathrm{P}=0.893)$, the fixed-effects model was applied when pooling HR. The synthesized results showed that patients with high $\mathrm{SUV}_{\text {mean }}$ had a shorter survival time than those with low $\mathrm{SUV}_{\text {mean }}$, but there was no statistical significance $(\mathrm{HR}=1.523,95 \% \mathrm{CI}=0.764-3.038, \mathrm{P}=0.232)($ Figure S4A) . Because of the limited data, publication bias and sensitivity analysis were not performed.

Two articles about TLG measurement of tumor tissue were included in the study. ${ }^{14,}{ }^{20}$ In the absence of heterogeneity $\left(\mathrm{I}^{2}=0.0 \%, \mathrm{P}=0.672\right)$, the fixed-effects model was applied when pooling HR. The synthesized results showed that patients with high TLG had a shorter survival time than those with low TLG, but the result had no statistical significance $(\mathrm{HR}=1.371,95 \% \mathrm{CI}=0.681-2.759, \mathrm{P}=0.377)$ (Figure S4B). Because of the limited data, publication bias and sensitivity analysis were not performed.

Two articles about nodal $\mathrm{SUV}_{\max }$ were included in the study. ${ }^{19,}{ }^{22}$ For no heterogeneity $\left(\mathrm{I}^{2}=54.1 \%, \mathrm{P}=\right.$ 0.140), the fixed-effects model was applied when pooling HR. The synthesized results showed that patients with high nodal $\mathrm{SUV}_{\max }$ had a shorter survival time than those with low nodal $\mathrm{SUV}_{\max }(\mathrm{HR}=2.095,95 \%$ $\mathrm{CI}=2.027-2.166, \mathrm{P}<0.001)$ (Figure $\mathbf{S 4 C}$ ) . Because of the limited data, publication bias and sensitivity analysis were not performed.

\section{Discussion}

In this meta-analysis, the results revealed that MTV and TLG measurement of tumor tissue showed potential as a biomarker for EFS. The results about primary tumor $\mathrm{SUV}_{\max }$ and nodal $\mathrm{SUV}_{\max }$ were significantly correlated with EFS with relatively high heterogeneity. Besides, primary tumor SUV $\max$ could be a biomarker for OS, and nodal $\mathrm{SUV}_{\max }$ was significantly related to OS with relatively high heterogeneity. Other FDG $\mathrm{PET} / \mathrm{CT}$ parameters were found to have no significant association with prognosis.

This meta-analysis focused on the FDG PET/CT parameters as a categorical variable, but the results of articles interesting in FDG PET/CT parameters as a continuous variable exhibited extreme discrepancy among different studies. For example, Carpenter et al. reported that primary tumor MTV and primary tumor TLG were significantly associated with OS in the univariate analyses, whereas only primary tumor TLG were significantly correlated with OS in the multivariate analyses. ${ }^{26}$ Calles-Sastre et al. found that primary-tumor MTV and primary-tumor TLG showed a significant association with OS and with RFS in the univariate analyses. ${ }^{27}$ Scher et al. detected primary-tumor MTV and primary-tumor TLG were correlated with OS and DFS in the univariate analyses, whereas primary-tumor TLG was the only FDG PET parameter significantly correlated with OS and DFS in the multivariate analyses. ${ }^{24}$ In addition, Liu et al. confirmed that primary-tumor MTV was a significant predictor of OS in the univariate analyses. ${ }^{28}$ Chong et al. reported that primary-tumor $\mathrm{SUV}_{\max }$, primary tumor MTV, and primary tumor TLG were significant prognostic factors for DFS in univariate analysis, but none of them was significant in the multivariate analyses (Table S2).$^{23}$

Apart from pretreatment FDG PET/CT parameters, some studies focused on the prognostic value of duringtreatment FDG PET/CT parameters. Carpenter et al. reported that primary-tumor MTV and primary- 
tumor TLG during treatment were significantly associated with OS and DFS. ${ }^{26}$ Liu et al. found that primary-tumor MTV during treatment was significantly associated with OS. ${ }^{28}$ Krhili et al. confirmed that not only primary-tumor MTV and primary-tumor TLG but also $\mathrm{SUV}_{\max }$ during treatment were significant prognostic factors for OS and DFS (Table S3).$^{29}$ Besides, changes between pre- and intra-treatment FDGPET/CT parameters also were confirmed to be prognostic factors in some studies. Park et al. confirmed that the percentage changes of $\mathrm{SUV}_{\max }$ have a prognostic value for predicting DFS. ${ }^{30} \mathrm{Oh}$ et al. showed that a decrease of SUV $\max$ was a statistically significant predictor of PFS. ${ }^{31}$ However, Carpenter et al. reported no correlation among the changes in primary-tumor $\mathrm{SUV}_{\max }, \mathrm{SUV}_{\text {mean }}$, MTV, TLG, and survival. ${ }^{26}$

Except for the pretreatment FDG PET/CT parameter, during-treatment FDG PET/CT parameter, and changes between pre- and intra-treatment FDG-PET/CT parameters, the one-two punch, using various types of prognostic factors to create a prognosis-predicting model, was a new orientation. For example, Hong et al. suggested a simple prognosis prediction model, using pretreatment FDG PET/CT and human papillomavirus (HPV) genotyping in patients with LACC treated with CCRT. ${ }^{32}$ Lee et al. constructed a nomogram based on these six (including age, nodal $\mathrm{SUV}_{\max }$, primary-tumor $\mathrm{SUV}_{\max }$, size, stage, and $\mathrm{SCC}$ ) and seven (including age, nodal $\mathrm{SUV}_{\max }$, primary-tumor $\mathrm{SUV}_{\max }$, size, stage, SCC, and high-risk HPV status) independent risk factors for two-year DFS and five-year OS. ${ }^{19}$ Besides, radiomics is a new frontier to predict the prognosis of patients with LACC. For example, Lucia et al. found that in LACC treated with CCRT, radiomics features such as Grey Level Non-uniformity GLRLM from PET are independent predictors of recurrence and loco-regional control with significantly higher prognostic power than usual clinical parameters. ${ }^{16}$ The predictive tool-enrolled FDG PET/CT parameter still needs more study.

Our study has some noteworthy limitations. First, all studies enrolled in the meta-analysis were retrospective, which might add selective bias. Second, significant heterogeneity existed in the results of this meta-analysis, and no subgroup analysis was performed for the limited studies. The potential resource of the heterogeneity was probably from four aspects. Patients in each study had different FIGO stages, different histologic subtypes, different treatment strategies, and different follow-up endpoints (Table 1-3) . Besides this, the different PET/CT technique and image analysis were existed in each study, which might cause the heterogeneity in this analysis (Table S4) . Third, the cut-off values for FDG PET/CT metabolic parameters were different in each study. Last, the HR used in this meta-analysis was directly extracted from a Cox proportional hazards model (multivariate analysis and univariate analysis) or indirectly estimated from Kaplan-Meier curves. By ignoring this discrepancy, the potential heterogeneity was exposed after synthesizing the results of all included studies. Considering these limitations, future large-scale prospective studies are needed to confirm the prognostic values of FDG PET metabolic parameters further. Despite these limitations, our study is the first meta-analysis to evaluate the prognostic value of FDG PET/CT metabolic parameters in LACC treated with CCRT and offers some significant findings.

\section{Disclosure of interests}

None declared. Completed disclosure of interest forms are available to view online as supporting information.

\section{Contributions to authorship}

QLL and YLZ conceived and designed the study. LH and QW extracted and analysed data. LH, LBZ and QW were the statisticians who confirmed the analysis of this study. LH, KLZ and YRW wrote the manuscript. QLL, YLZ and XF critically revised the manuscript. All authors reviewed and approved the final manuscript.

\section{Details of ethics approval}

Not applicable.

\section{Funding}

This work was supported by the Natural Science Basic Research Program of Shaanxi (2020JQ-952), This work was supported by the Natural Science Basic Research Program of Shaanxi (2017ZDJC-11, 2018JM7073), the 
Clinical Research Award of the First Affiliated Hospital of Xi'an Jiaotong University, China (XJTU1AF2018-017, XJTU1AF-CRF-2019-002). The funders had no role in study design, data collection and analysis, decision to publish, or preparation of the manuscript.

\section{Acknowledgements}

None.

\section{Supporting Information}

Additional supporting information may be found online in the Supporting Information section at the end of the article.

Figure S1. Results for primary SUVmax for DFS. (A) Forest plots of the HRs for SUVmax. (B) Begg's funnel plot for SUVmax. (C) Sensitivity analysis of the influence of each individual study on the pooled HR for SUVmax.

Figure S2. Results for nodal SUVmax for DFS. (A) Forest plots of the HRs. (B) Begg's funnel plot. (C) Sensitivity analysis of the influence of each individual study on the pooled HR.

Figure S3. Results for primary SUVmax for OS. (A) Forest plots of the HRs. (B) Begg's funnel plot. (C) Sensitive analysis of the influence of each individual study on the pooled HR.

Figure S4. Results for primary SUVmean, TLG and nodal SUVmax, and for OS. (A) Forest plots of the HRs with primary SUVmean. (B) Forest plots of the HRs with primary TLG. (C) Forest plots of the HRs with nodal SUVmax.

Table S1. Searching strategy.

Table S2. Summary of prognostic results about pretreatment FDG PET parameters as a continuous variable

Table S3. Summary of prognostic results about FDG PET parameters during treatment.

Table S4. Information about PET/CT technique and image analysis

\section{Reference}

1. Siegel RL, Miller KD, Jemal A. Cancer statistics, 2020. CA Cancer J Clin. 2020 Jan;70(1):7-30.

2. Green JA, Kirwan JM, Tierney JF, Symonds P, Fresco L, Collingwood M, et al. Survival and recurrence after concomitant chemotherapy and radiotherapy for cancer of the uterine cervix: a systematic review and meta-analysis. Lancet. 2001 Sep 8;358(9284):781-6.

3. Endo D, Todo Y, Okamoto K, Minobe S, Kato H, Nishiyama N. Prognostic factors for patients with cervical cancer treated with concurrent chemoradiotherapy: a retrospective analysis in a Japanese cohort. J Gynecol Oncol. 2015 Jan;26(1):12-8.

4. Chemoradiotherapy for Cervical Cancer Meta-Analysis C. Reducing uncertainties about the effects of chemoradiotherapy for cervical cancer: a systematic review and meta-analysis of individual patient data from 18 randomized trials. J Clin Oncol. 2008 Dec 10;26(35):5802-12.

5. Li X, Wei LC, Zhang Y, Zhao LN, Li WW, Ping LJ, et al. The Prognosis and Risk Stratification Based on Pelvic Lymph Node Characteristics in Patients With Locally Advanced Cervical Squamous Cell Carcinoma Treated With Concurrent Chemoradiotherapy. Int J Gynecol Cancer. 2016 Oct;26(8):1472-9.

6. Chen CC, Wang L, Lin JC, Jan JS. The prognostic factors for locally advanced cervical cancer patients treated by intensity-modulated radiation therapy with concurrent chemotherapy. J Formos Med Assoc. 2015 Mar;114(3):231-7. 
7. Teh J, Yap SP, Tham I, Sethi VK, Chua EJ, Yeo R, et al. Concurrent chemoradiotherapy incorporating high-dose rate brachytherapy for locally advanced cervical carcinoma: survival outcomes, patterns of failure, and prognostic factors. Int J Gynecol Cancer. 2010 Apr;20(3):428-33.

8. Herrera FG, Prior JO. The role of PET/CT in cervical cancer. Front Oncol. 2013;3:34.

9. Guo F, Yang R, Tian J, Fan L. The role of F-fluorodeoxyglucose (FDG) positron emission tomographycomputed tomography $(\mathrm{PET} / \mathrm{CT})$ in the screening of cervical cancer: a literature review. Cell Biochem Biophys. 2014 Jun;69(2):197-201.

10. N G, MA A, WE P, AG R, TD B. - FDG-PET Imaging in Cervical Cancer. D - 1264464. (- 1558-4623 (Electronic)):- 461-70.

11. Im HJ, Yoon HJ, Lee ES, Kim TS, Kim JY, Chung JK, et al. Prognostic implication of retrocrural lymph node involvement revealed by (18)F-FDG PET/CT in patients with uterine cervical cancer. Nucl Med Commun. 2014 Mar;35(3):268-75.

12. Chong GO, Jeong SY, Park SH, Lee YH, Lee SW, Hong DG, et al. Comparison of the Prognostic Value of F-18 Pet Metabolic Parameters of Primary Tumors and Regional Lymph Nodes in Patients with Locally Advanced Cervical Cancer Who Are Treated with Concurrent Chemoradiotherapy. PLoS One. 2015;10(9):e0137743.

13. Akkus Yildirim B, Onal C, Erbay G, Cem Guler O, Karadeli E, Reyhan M, et al. Prognostic values of ADCmean and SUVmax of the primary tumour in cervical cancer patients treated with definitive chemoradiotherapy. J Obstet Gynaecol. 2019 Feb;39(2):224-30.

14. Cima S, Perrone AM, Castellucci P, Macchia G, Buwenge M, Cammelli S, et al. Prognostic Impact of Pretreatment Fluorodeoxyglucose Positron Emission Tomography/Computed Tomography SUVmax in Patients With Locally Advanced Cervical Cancer. Int J Gynecol Cancer. 2018 Mar;28(3):575-80.

15. Guler OC, Torun N, Yildirim BA, Onal C. Pretreatment metabolic tumour volume and total lesion glycolysis are not independent prognosticators for locally advanced cervical cancer patients treated with chemoradiotherapy. Br J Radiol. 2018 Apr;91(1084):20170552.

16. Lucia F, Visvikis D, Desseroit MC, Miranda O, Malhaire JP, Robin P, et al. Prediction of outcome using pretreatment (18)F-FDG PET/CT and MRI radiomics in locally advanced cervical cancer treated with chemoradiotherapy. Eur J Nucl Med Mol Imaging. 2018 May;45(5):768-86.

17. Hong JH, Jung US, Min KJ, Lee JK, Kim S, Eo JS. Prognostic value of total lesion glycolysis measured by 18F-FDG PET/CT in patients with locally advanced cervical cancer. Nucl Med Commun. 2016 Aug;37(8):843-8.

18. Onal C, Reyhan M, Parlak C, Guler OC, Oymak E. Prognostic value of pretreatment 18Ffluorodeoxyglucose uptake in patients with cervical cancer treated with definitive chemoradiotherapy. Int $\mathrm{J}$ Gynecol Cancer. 2013 Jul;23(6):1104-10.

19. Lee WK, Chong GO, Jeong SY, Lee HJ, Park SH, Ryu JM, et al. Prognosis-Predicting Model Based on [(18)F]fluorodeoxyglucose PET Metabolic Parameters in Locally Advanced Cervical Cancer Patients Treated with Concurrent Chemoradiotherapy: Multi-Center Retrospective Study. J Clin Med. 2020 Feb 5;9(2).

20. Herrera FG, Breuneval T, Prior JO, Bourhis J, Ozsahin M. [(18)F]FDG-PET/CT metabolic parameters as useful prognostic factors in cervical cancer patients treated with chemo-radiotherapy. Radiat Oncol. 2016 Mar 16;11:43.

21. Yilmaz B, Dag S, Ergul N, Cermik TF. The efficacy of pretreatment and after treatment 18F-FDG $\mathrm{PET} / \mathrm{CT}$ metabolic parameters in patients with locally advanced squamous cell cervical cancer. Nucl Med Commun. 2019 Mar;40(3):219-27. 
22. Onal C, Guler OC, Reyhan M, Yapar AF. Prognostic value of 18F-fluorodeoxyglucose uptake in pelvic lymph nodes in patients with cervical cancer treated with definitive chemoradiotherapy. Gynecol Oncol. 2015 Apr;137(1):40-6.

23. Chong GO, Lee WK, Jeong SY, Park SH, Lee YH, Lee SW, et al. Prognostic value of intratumoral metabolic heterogeneity on F-18 fluorodeoxyglucose positron emission tomography/computed tomography in locally advanced cervical cancer patients treated with concurrent chemoradiotherapy. Oncotarget. 2017 Oct 27;8(52):90402-12.

24. Scher N, Castelli J, Depeursinge A, Bourhis J, Prior JO, Herrera FG, et al. ((18)F)-FDG PET/CT parameters to predict survival and recurrence in patients with locally advanced cervical cancer treated with chemoradiotherapy. Cancer Radiother. 2018 May;22(3):229-35.

25. Vercellino L, Groheux D, Thoury A, Delord M, Schlageter MH, Delpech Y, et al. Hypoxia imaging of uterine cervix carcinoma with (18)F-FETNIM PET/CT. Clin Nucl Med. 2012 Nov;37(11):1065-8.

26. Carpenter DJ, Jacobs CD, Wong TZ, Craciunescu O, Chino JP. Changes on Midchemoradiation Therapy Fluorodeoxyglucose Positron Emission Tomography for Cervical Cancer Are Associated with Prognosis. Int J Radiat Oncol Biol Phys. 2019 Oct 1;105(2):356-66.

27. Calles-Sastre L, Mucientes-Rasilla J, San-Frutos Llorente LM, Royuela A, Garcia-Espantaleon Navas M, Herrero Gamiz S, et al. Prognostic significance of metabolic tumor volume and total lesion glycolysis in patients with advanced cervical carcinoma. Rev Esp Med Nucl Imagen Mol. 2019 Jan - Feb;38(1):17-21.

28. Liu FY, Lai CH, Yang LY, Wang CC, Lin G, Chang CJ, et al. Utility of (18)F-FDG PET/CT in patients with advanced squamous cell carcinoma of the uterine cervix receiving concurrent chemoradiotherapy: a parallel study of a prospective randomized trial. Eur J Nucl Med Mol Imaging. 2016 Sep;43(10):1812-23.

29. Krhili S, Muratet JP, Roche S, Pointreau Y, Yossi S, Septans AL, et al. Use of Metabolic Parameters as Prognostic Factors During Concomitant Chemoradiotherapy for Locally Advanced Cervical Cancer. Am J Clin Oncol. 2017 Jun;40(3):250-5.

30. Park JJ, Kim CK, Park BK. Prognostic value of diffusion-weighted magnetic resonance imaging and (18)F-fluorodeoxyglucose-positron emission tomography/computed tomography after concurrent chemoradiotherapy in uterine cervical cancer. Radiother Oncol. 2016 Sep;120(3):507-11.

31. Oh D, Lee JE, Huh SJ, Park W, Nam H, Choi JY, et al. Prognostic significance of tumor response as assessed by sequential 18F-fluorodeoxyglucose-positron emission tomography/computed tomography during concurrent chemoradiation therapy for cervical cancer. Int J Radiat Oncol Biol Phys. 2013 Nov 1;87(3):54954.

32. Hong CM, Park SH, Chong GO, Lee YH, Jeong JH, Lee SW, et al. Enhancing prognosis prediction using pre-treatment nodal SUVmax and HPV status in cervical squamous cell carcinoma. Cancer Imaging. 2019 Jun 24;19(1):43.

Figure legends

Figure 1. Flow chart of the literature search.

Figure 2. Results for primary MTV and TLG for DFS (A) Forest plots of the HRs for DFS with MTV. (B) Forest plots of the HRs for DFS with TLG. (C) Begg's funnel plot for TLG. (D) Sensitivity analysis of the influence of each individual study on the pooled HR for TLG.

Figure 3. Results for primary $\mathbf{S U V}_{\max }$ for OS.(A) Forest plots of the HRs. (B) Begg's funnel plot. (C) Sensitivity analysis of the influence of each individual study on the pooled HR.

Table 1. Basic information and patient characteristics. 


\begin{tabular}{lllllllll}
\hline Surname of first author & Year & Country & Study design & No. of patients & Age & Age & Histology & Histology \\
\hline & & & & & Median & Range & SSC & ACa \\
Yilmaz & 2018 & Turkey & Retrospective & 44 & 54.6 & $28-78$ & NS & NS \\
Yildirim & 2018 & Turkey & Retrospective & 63 & 58 & $21-86$ & NS & NS \\
Cima & 2017 & Switzerland & Retrospective & 92 & 57 & $30-89$ & 71 & 14 \\
Guler & 2018 & Turkey & Retrospective & 129 & 57 & $22-83$ & 119 & 10 \\
Lucia & 2018 & France & Retrospective & 69 & 58 & $29-90$ & 54 & 9 \\
Hong & 2016 & Korea & Retrospective & 56 & 57 & $32-81$ & 49 & 7 \\
Herrera & 2016 & Switzerland & Retrospective & 38 & 52.5 & $26-83$ & 33 & 5 \\
Chong & 2015 & Korea & Retrospective & 56 & 51.5 & NS & 50 & 6 \\
Onal & 2015 & Korea & Retrospective & 93 & 58 & $30-89$ & 87 & 6 \\
Im & 2014 & Korea & Retrospective & 145 & 55 & $22-88$ & 131 & 10 \\
Onal & 2013 & Turkey & Retrospective & 149 & 58 & $21-86$ & 138 & 11 \\
Chong & 2017 & Korea & Retrospective & 93 & 53.1 & NS & 85 & 5 \\
Lee & 2020 & Korea & Retrospective & 270 & NS & NS & 243 & NS \\
Vercellino & 2012 & France & Retrospective & 16 & 57 & $32-69$ & 15 & 1 \\
\hline
\end{tabular}

SSC, squamous cell carcinoma; AC, adenocarcinoma, NS, not stated

Table 2 Disease features: stage and follow-up

\begin{tabular}{|c|c|c|c|c|c|c|c|}
\hline $\begin{array}{l}\text { Surname } \\
\text { of first } \\
\text { au- } \\
\text { thor }\end{array}$ & \multirow{2}{*}{$\begin{array}{l}\text { Stage } \\
\text { IB }\end{array}$} & \multirow{2}{*}{$\begin{array}{l}\text { Stage } \\
\text { IIA }\end{array}$} & \multirow{2}{*}{$\begin{array}{l}\text { Stage } \\
\text { IIB }\end{array}$} & \multirow{2}{*}{$\begin{array}{l}\text { Stage } \\
\text { IIIA }\end{array}$} & \multirow{2}{*}{$\begin{array}{l}\text { Stage } \\
\text { IIIB }\end{array}$} & \multirow{2}{*}{$\begin{array}{l}\text { Stage } \\
\text { IV }\end{array}$} & \multirow{2}{*}{$\begin{array}{l}\text { Follow- } \\
\text { up } \\
\text { Median }\end{array}$} \\
\hline & & & & & & & \\
\hline Yilmaz & 7 & 2 & 27 & 1 & 3 & 4 & 22 \\
\hline Yildirim & 4 & 4 & 38 & 8 & 9 & - & 60 \\
\hline Cima & - & - & 78 & 3 & 4 & 7 & 31 \\
\hline Guler & 16 & 4 & 52 & 13 & 36 & 8 & 30 \\
\hline Lucia & 10 & 4 & 36 & 1 & 9 & 9 & 36 \\
\hline Hong & - & - & 38 & 3 & 6 & 9 & NS \\
\hline Herrera & 6 & 10 & 15 & 2 & 4 & 1 & 52.5 \\
\hline $\begin{array}{l}\text { Chong } \\
(2015)\end{array}$ & - & - & 44 & 7 & 5 & - & 42 \\
\hline Onal & 9 & 2 & 49 & 10 & 21 & 2 & 29 \\
\hline $\operatorname{Im}$ & 19 & 8 & 90 & 5 & 20 & 3 & NS \\
\hline Onal & 16 & 7 & 84 & 15 & 24 & 3 & 29 \\
\hline $\begin{array}{l}\text { Chong } \\
(2017)\end{array}$ & - & - & 75 & 12 & 5 & 1 & 55 \\
\hline Lee & - & - & 215 & NS & NS & NS & 49.5 \\
\hline Vercellino & 1 & 1 & 8 & 3 & 3 & 3 & \\
\hline
\end{tabular}

NS, not stated

Table 3 Treatment-related features. 


\begin{tabular}{|c|c|c|c|c|c|c|}
\hline $\begin{array}{l}\text { Surname } \\
\text { of first } \\
\text { author }\end{array}$ & EBRT & EBRT & EBRT & ICR & ICR & ICR \\
\hline & Type & $\begin{array}{l}\text { Total } \\
\text { dose Me- } \\
\text { dian(range) }\end{array}$ & $\begin{array}{l}\text { Fraction } \\
\text { (Gy) }\end{array}$ & $\begin{array}{l}\text { Fraction } \\
\text { (Gy) }\end{array}$ & Number & Number \\
\hline Yilmaz & $\begin{array}{l}\text { 3D- } \\
\text { CRT }\end{array}$ & 50.4 & 1.8 & 6 & 6 & 5 \\
\hline Yildirim & $\begin{array}{l}\text { 3D- } \\
\text { CRT }\end{array}$ & 50.4 & 1.8 & 7 & 7 & 4 \\
\hline Cima & $\begin{array}{l}\text { 3D- } \\
\text { CRT }\end{array}$ & NS & NS & NS & NS & NS \\
\hline Guler & $\begin{array}{l}\text { 3D- } \\
\text { CRT }\end{array}$ & 50.4 & 1.8 & 7 & 7 & 4 \\
\hline Lucia & $\begin{array}{l}\text { 3D- } \\
\text { CRT/IMRT }\end{array}$ & $45-50.4$ & NS & 7 & 7 & $3-4$ \\
\hline Hong & NS & 54 & 2 & 3.5 & 3.5 & 8 \\
\hline Herrera & IGRT & $\begin{array}{l}45(45- \\
50.4)\end{array}$ & NS & 7 & 7 & $3-4$ \\
\hline $\begin{array}{l}\text { Chong } \\
(2015)\end{array}$ & 3D-CRT & 45 & 1.8 & - & - & - \\
\hline Onal & $\begin{array}{l}\text { 3D- } \\
\text { CRT }\end{array}$ & 50.4 & 1.8 & 7 & 7 & 4 \\
\hline $\operatorname{Im}$ & NS & NS & NS & NS & NS & NS \\
\hline
\end{tabular}


Surname

of first

author

Onal

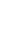

EBRT

3D-

CRT

Chong

3D-CRT

(2017)

Lee

3D-

CRT

Vercellino

NS
45

1.8

1.8

45

EBRT

50.4

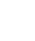

1.8

EBRT

7

$\frac{\text { ICR }}{7}$

R

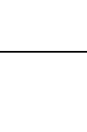

ICR

7

6

NS

NS

6

NS

NS

EBRT, External Beam Radiotherapy; ICR Intracavitary Brachytherapy IGRT, Image Guided Radiation Therapy; 3D-CRT, 3D Conformal Therapy; IMRT, Intensity-Modulated Radiation Therapy; NS, not stated

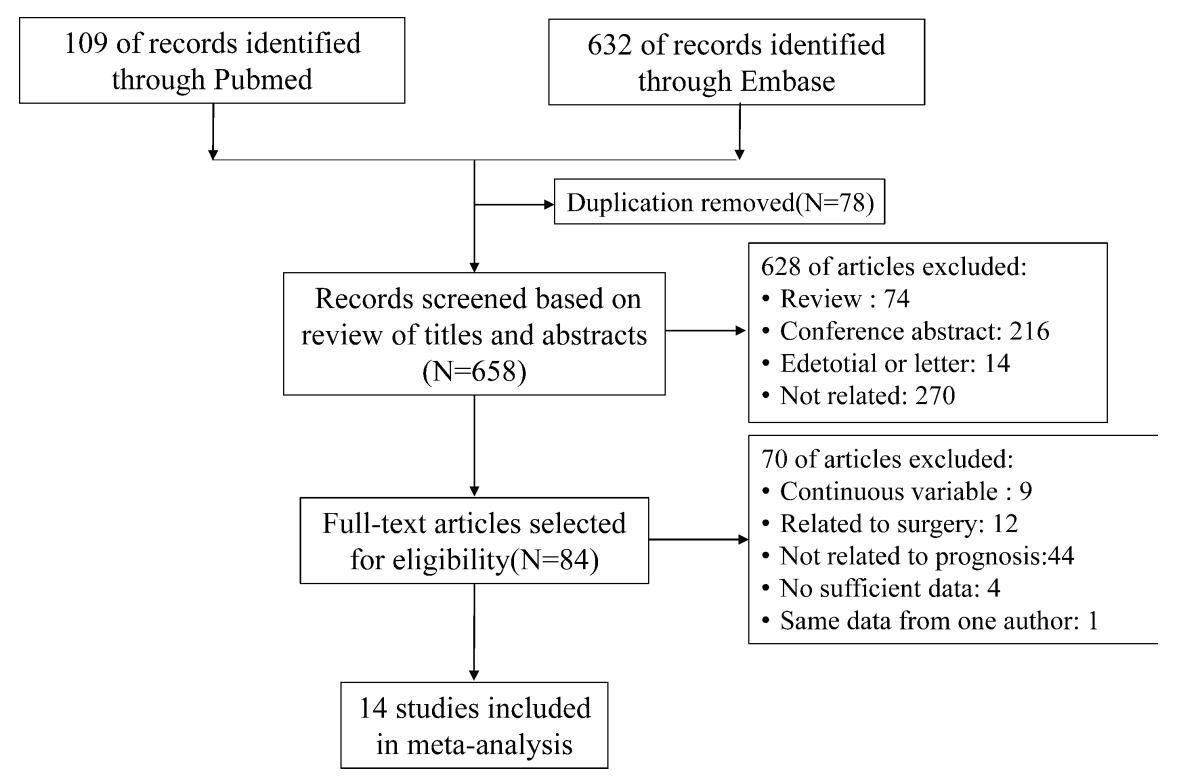

Cc

ch ap

W

40

$\mathrm{m}$

$$
\text { cis }
$$

\section{W}

40

cis

$\mathrm{W}$
40 


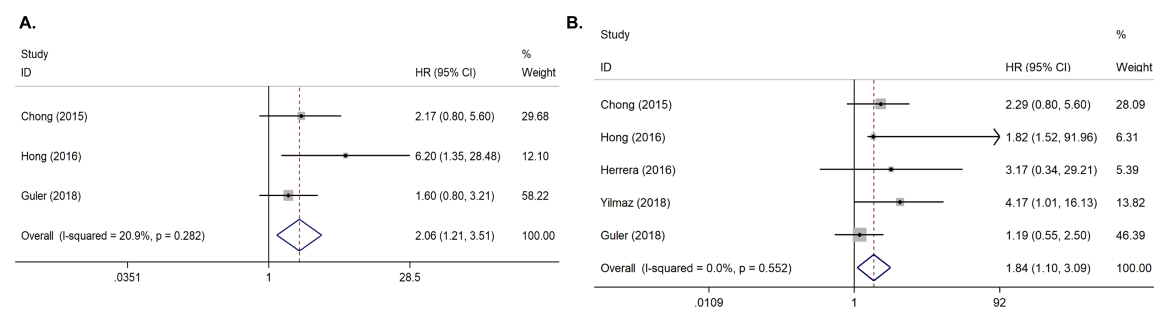

c.

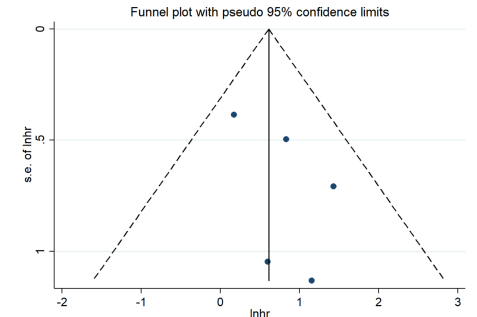

D.

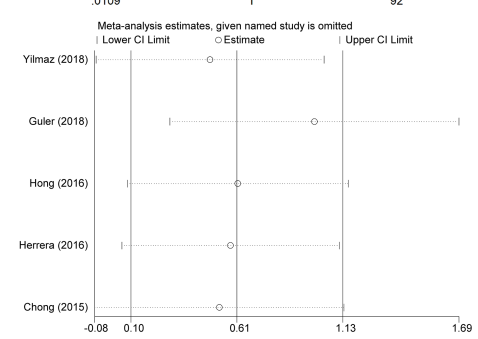

A. Stud

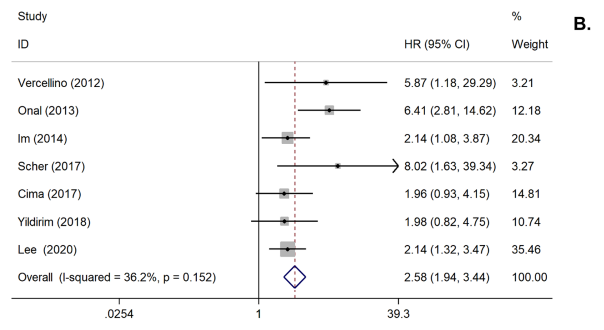

B.

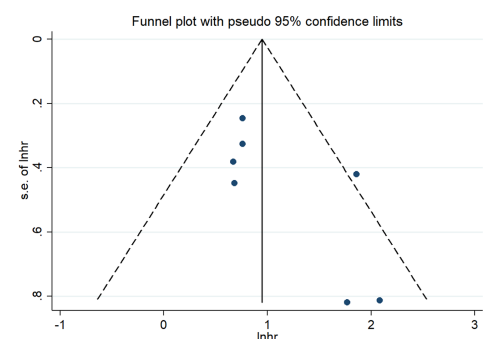

c.

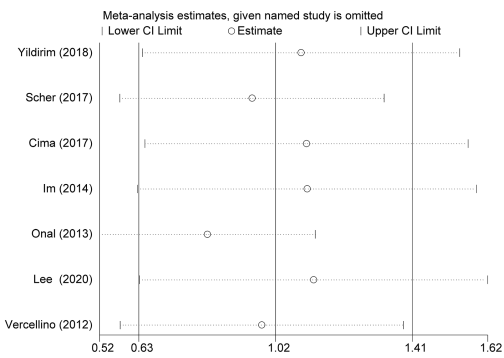

